\title{
Variable camber airfoil: New concept, new challenge
}

Aircraft morphing, especially in the case of the variable camber airfoil, has potential from the viewpoints of both fundamental aerodynamics and flight application. In the field of aerodynamics, the use of a variable camber airfoil can improve aircraft flight performance and allow efficient multiple missions; in biology, the concept of the variable camber airfoil can be employed to explain the mystery of animal flight.

In the case of low-speed aircraft, the performance of low-Reynolds-number airfoils is greatly determined by the relatively feeble separation resistance of the laminar boundary layer. Better understanding and effective control of laminar separation is therefore valuable for improving the flight mechanics of low-Reynolds-number aircraft such as micro air vehicles (MAVs). Recent research has demonstrated that the variable camber airfoil has obvious advantages in terms of the control of separation and flight maneuverability. Furthermore, compliant deformation of the airfoil helps explain the nature of the aerodynamic characteristics of a membrane wing such as that of a bat. However, little attention has been paid to the quasi-steady aerodynamic characteristics resulting from the camber deformation and the effect of different initial flow conditions. Prof. Yang JiMing and his group at the University of Science and Technology of China set out to explore this area of research. Through aerodynamic load measurements and related flow visualization, they distinguished the aerodynamic characteristics of the variable camber airfoil for steady and quasi-steady flow separation behaviors. Their work, entitled "Experimental investigation on the quasi-steady flow separation behaviors of a variable camber wing", was published in Scientia Sinica Physica, Mechanica \& Astronomica, 2012, Vol. 42(5).

The group firstly designed a type of variable camber airfoil test model whose camber can be changed smoothly. The baseline airfoil is assumed to have an NACA 0015 profile. A rigid D-spar, the primary load carrying member, extends from the leading edge of the airfoil to the 30-percent chord. The D-spar does not undergo any deformation in the chordwise direction, and consequently, only the section aft of the D-spar can deform. This section aft of the spar is specially designed to have an internal substructure. The substructure comprises five rows of hinge-like units ranked serially along the chordwise direction. The substructure is designed to support a flexible skin that provides the aerodynamic shape and is capable of morphing. A servomotor was fixed in the D-spar to provide power to pull the lower surface of the aft section into the D-spar to a certain extent with the help of gearing. As part of the lower surface is pulled into the D-spar, there is a discrepancy in the area between the upper surface and lower surface and the airfoil is thus asymmetric; in other words, the airfoil has some camber. The D-spar was made with acrylic materials and the skin of the aft section was made with polypropylene plastics (Figure 1).

Aerodynamic load measurements and a flow visualization technique such as particle image velocimetry are combined to reveal the aerodynamic characteristics resulting from the camber deformation and corresponding flow structures, from which the complex mechanism is hopefully determined. Research shows that on the occasion of flow separation, different camber deformation paths have totally distinct aerodynamic characteristics. Prof. Yang's group found that the stall type determines the distinction. For some camber deformation paths, the stall is a type of leading-edge stall. During this stall process, flow separates rather suddenly and abruptly over the entire top surface of the airfoil, with the origin of this separation being at the leading edge. Therefore, the lift of the airfoil is rather low under the circumstances of large camber. However, for other camber deformation paths, the stall is a type of trailing-edge stall. For this type of stall, there is progressive and gradual movement of separation from the trailing edge toward the leading edge as the camber is increased. Because of this, the trailing-edge stall is "soft" compared with the leading-edge stall and the lift is higher under the same conditions.

Therefore, under flow-separation circumstances, the flow around the airfoil is related not only to the current environment but also to the previous flow characteristics, and the history of flow evolvement should thus be considered in the design of low-Reynolds-number aircraft. The role of the history of flow evolvement tends to be overlooked in traditional aircraft design. Designers often only focus on the steady aerodynamic characteristics of an aircraft. However, to further improve the flight performance of MAVs, engineers will have to face this new challenge.

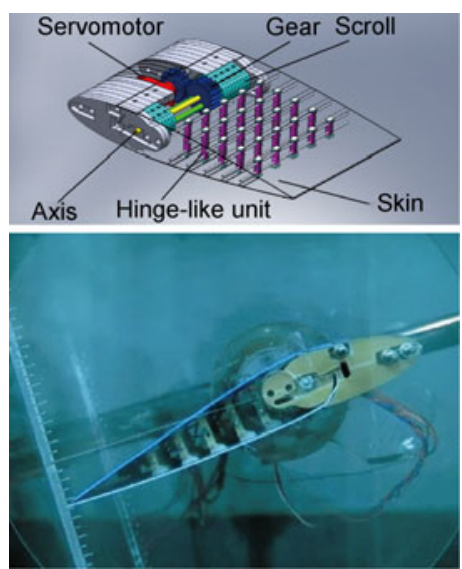

Figure 1 (Color online) Construction of a variable camber airfoil and a real model.

See the article: Yang W C, Yang J T, Wang J, et al. Experimental investigation on the quasi-steady flow separation behaviors of a variable camber wing (in Chinese). Sci Sin Phys Mech Astron, 2012, 42: 531-537

Open Access This article is distributed under the terms of the Creative Commons Attribution License which permits any use, distribution, and reproduction in any medium, provided the original author(s) and source are credited. 